\title{
Relative nonlinearity of BRIL luminometers derived from CMS $\mu$ scans
}

\section{A. Babaev* on behalf of the CMS Collaboration}

\author{
Tomsk Polytechnic University, Tomsk, Russia
}

E-mail: krass58addmai].ru

The Beam Radiation Instrumentation and Luminosity Project (BRIL) is devoted to the simulation and measurements of beam conditions and luminosity for CMS. One of the BRIL tasks is the luminosity monitoring and calibration of the detectors to obtain the luminosity. For precise calibration of the luminosity it is important to understand the influence of nonlinear effects on single bunch instantaneous luminosity evaluated from measurements of a luminometer. These proceedings present the method to estimate nonlinearity of a luminometer based on data from $\mu$ scans, which are beam scans performed at conditions optimized for nonlinearity studies.

7th Annual Conference on Large Hadron Collider Physics - LHCP2019

20-25 May, 2019

Puebla, Mexico

${ }^{*}$ Speaker. 


\section{Introduction}

BRIL (Beam Radiation Instrumentation and Luminosity) is a project of the CMS experiment [W] at CERN. The project is tasked with simulation and measurements of luminosity as well as beam conditions and radiation fields in CMS. BRIL is engaged in developing new detectors, compatible with the high luminosity experimental environments at the LHC. BRIL operates several detectors based on different physical principles and technologies. In this proceedings the hadron forward (HF) calorimeters [ [D, [3] and the Fast Beam Conditions Monitor (BCM1F) [四] will be considered. HF detects Cherenkov radiation in quartz fibers. It is installed at $11.2 \mathrm{~m}$ away from the interaction point on both sides. There are two types of luminosity determination based on HF: the occupancy counting (HFOC) and the transverse energy sum (HFET) methods. BCM1F is a fast particle counting detector using crystal silicon and diamond sensors. The sensors are arranged in two rings around the beam pipe at $1.8 \mathrm{~m}$ on both sides from the interaction point. This detector is sensitive to both collisions products and beam background because of its high time resolution. In the proceedings the notations PCVD and SI refer to luminosity provided by policrystalline diamond based sensors and by silicon based sensors, correspondingly.

Also, data from the CMS pixel detector are used in luminosity measurements. In the pixel cluster counting (PCC) method, luminosity is evaluated from the average number of pixel clusters in zero-bias events [వ]].

Measured raw numbers (referred to as "rates" below) provided by luminometers cannot be used interchangeably because of the different counting methods. Detector rates increase with pileup (number of proton-proton interactions resulting from a single bunch collision). Measured rates are translated to luminosity by using a specific calibration procedure. The calibration provides the detector-independent quantity — luminosity [15, 目]. For a pair of colliding bunches, the single bunch instantaneous luminosity (SBIL) is defined as:

$$
\mathrm{SBIL}=\frac{f_{\mathrm{rev}} R}{\sigma_{\mathrm{vis}}},
$$

where $f_{\text {rev }}$ is the LHC revolution frequency and $\sigma_{\text {vis }}$ is the calibration constant corresponding to the luminometer. The latter measures rates $R$.

A beam scan is an experiment that allows varying the pileup (and, consequently, rates) by changing the separation between orbits of the two colliding beams. The overlap of two colliding beams is minimal at large beam separation and maximal at head-on collisions. As a result, the measured rates are changed correspondingly to the varying of beam separation during the scan. Beam scans are performed at different settings for the variety of goals, in particular van der Meer scan for absolute calibration of luminometers (where calibration constant $\sigma_{\mathrm{vis}}$ is derived [ 6 , []]). The $\mu$ scans are another type of beam scans performed at the CMS especially for nonlinearity studies. While the beam separation is changed $\mu$ scans cover a range of pileup from about 50 to less than 1 . The $\mu$ scans are performed with standard beam optics of $\beta^{*}=30 \mathrm{~cm}$ and beam transverse RMS size $(\sigma)$ of about $15 \mu \mathrm{m}$ (i.e., beam overlap width less than $20 \mu \mathrm{m}$ ). The beam separation varies from zero at head-on collisions to $5 \sigma$ where the beam overlap is minimal providing the least pileup. The $\mu$ scans considered in this paper were performed with 15 steps and the beam separation changed from $5 \sigma$ to head-on collisions and then to $5 \sigma$ again. The waiting time per step was $47 \mathrm{~s}$ providing 
sufficient statistical precision for nonlinearity studies. As an example, the change of rates during the $\mu$ scans performed in LHC fill $6854^{1}$ is shown in Fig. 1 .

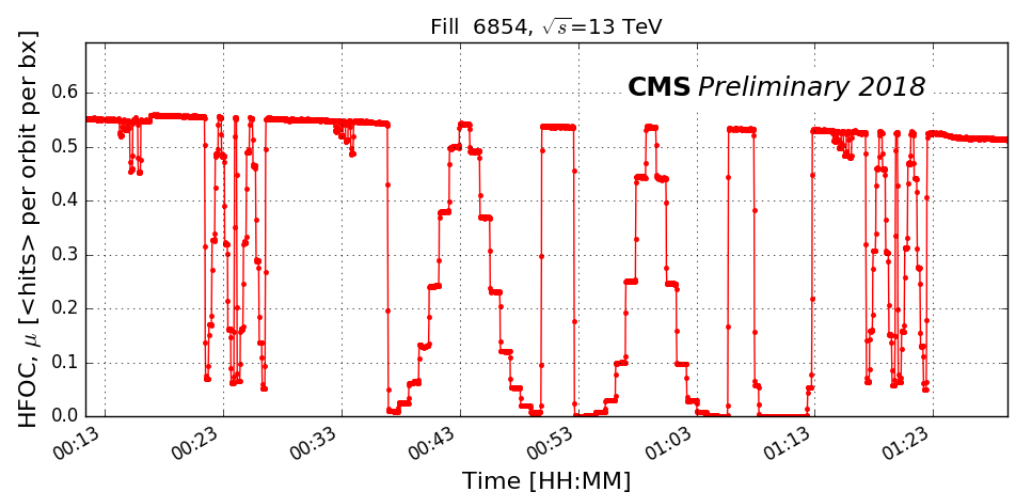

Figure 1: HFOC rates evolution during LHC fill 6854. Two $\mu$ scans were between 00:35 and 01:05 (2018, June 28).

An ideal luminometer response (measured rates) is linearly proportional to pileup. But real detectors, because of various detector effects, exhibit nonlinear behavior, which has to be understood and estimated. The response of a luminometer could be nonlinear function of pileup due to ratedependent inefficiency, signal spillover from one bunch collision time window to the next, etc. The focus in these proceedings is on the consistency of luminosity provided by different luminometers. The relative behavior of luminosity provided by different methods is compared while the pileup is changed during a $\mu$ scan. The term "relative nonlinearity" is used to describe that behavior. The ideal luminometer should have linear rate dependence on pileup. If this ideal linear luminometer is known from some physical considerations then one can compare other luminometers with that linear luminometer by using the relative nonlinearity method, and the deflection from linear response might be revealed.

\section{The concept of relative nonlinearity}

The relative nonlinearity defines the behavior of luminosity measured by the luminometer $\mathrm{L}$ in relation to the luminosity measured by the reference luminometer $\mathrm{L}_{\text {ref }}$. The luminosity measured by different methods can be compared because SBIL is calibrated as defined by $(\mathbb{L}$. ratio

$$
\text { Ratio }=\frac{\operatorname{SBIL}(\mathrm{L})}{\operatorname{SBIL}\left(\mathrm{L}_{\mathrm{ref}}\right)}
$$

is studied within the range of pileup covered during the $\mu$ scan. If luminometer $\mathrm{L}_{\text {ref }}$ is close to ideal linear luminometer and it has negligible background, then $\operatorname{SBIL}\left(\mathrm{L}_{\mathrm{ref}}\right)=b \mu$ where $b$ is a constant and $\mu$ is the pileup. For the test luminometer L, in general case, SBIL(L) exhibits higher orders of

\footnotetext{
${ }^{1}$ LHC fill is the LHC operational cycle that includes setup, injection, ramp, adjustment, stable beam phase and beam dump. It is assigned an incremental fill number [ $[\mathbb{}]$. During the beam stable phase LHC beams are set in collisions.
} 
$\mu$. Therefore, the ratio (لd ) can be considered as a function of $\operatorname{SBIL}\left(\mathrm{L}_{\text {ref }}\right)$. Then, the ratio (L. fitted with a first-order polynomial

$$
\text { Ratio }=p_{0}+p_{1} \times \operatorname{SBIL}\left(\mathrm{L}_{\mathrm{ref}}\right),
$$

which represents the behavior well within the available measurement precision. The slope $p_{1}$ is used as the quantitative characteristic of luminometers' relative nonlinearity as shown in Fig. $\square$. The parameter $p_{1}=0$ corresponds to the fact that test luminometer $\mathrm{L}$ is close to ideal linear luminometer. Values of $p_{1}$ different than zero indicate a nonlinear relative behavior of luminosity measured by luminometers $\mathrm{L}$ and $\mathrm{L}_{\mathrm{ref}}$. Below the term "nonlinearity" will refer to the parameter $p_{1}$. The parameter $p_{0}$ is close to 1 because it corresponds to the low-SBIL limit at which the absolute calibration [ [ $[$, 目] is performed and hence good agreement of SBIL provided by all luminometers is expected. To minimize the influence from machine induced background and noise only the range where $\operatorname{SBIL}\left(\mathrm{L}_{\mathrm{ref}}\right)>1 \mathrm{~Hz} / \mu \mathrm{b}$ is considered.

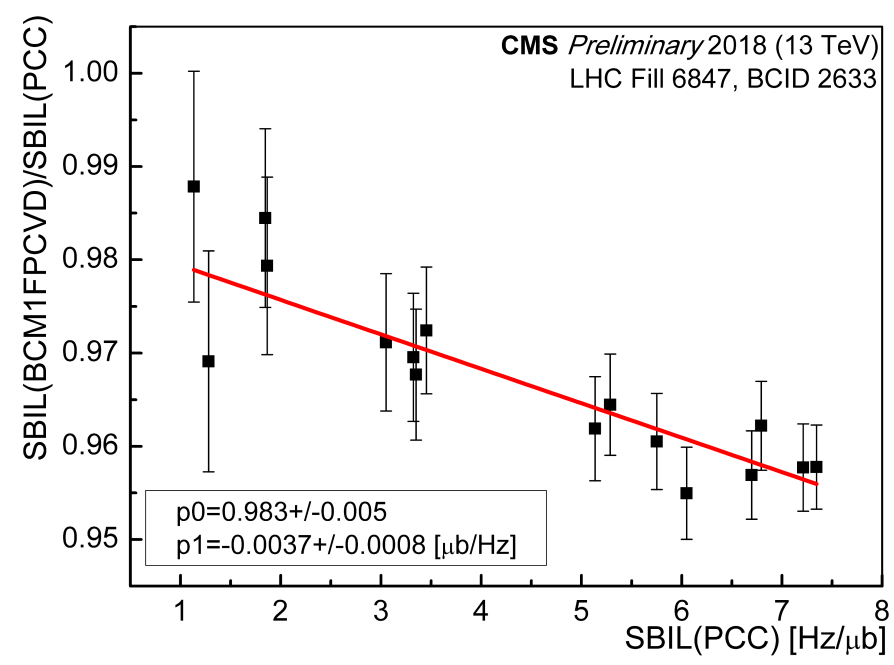

Figure 2: Sample of the fit using the data for bunch crossing ID (BCID, see footnote 3 below) 2633, $\mu$ scan performed in LHC fill 6847. Black points are the ratios (D. 1 ) SBIL(BCM1FPCVD)/SBIL(PCC) calculated using measured rates. The red line is the fit (2.2) when the ratio SBIL(BCM1FPCVD)/SBIL(PCC) is considered in dependence on SBIL(PCC). Fit parameters are shown in the legend.

The reference scale $\operatorname{SBIL}\left(\mathrm{L}_{\text {ref }}\right)$ is chosen to have good time stability and small nonlinear dependence on pileup. Where possible, PCC data ${ }^{2}$ are used as $\operatorname{SBIL}\left(\mathrm{L}_{\mathrm{ref}}\right)$.

\section{Relative nonlinearity of BRIL luminometers}

The method for nonlinearity studies presented here requires the choice of the best reference scale from additional physical considerations. Usually PCC data with sufficient statistical precision

\footnotetext{
${ }^{2}$ The probability of a given pixel being hit by two different particles from the same bunch crossing is exceedingly small. Therefore, the number of hit pixel clusters per crossing is expected to be a linear function of the number of interactions per crossing [圆].
} 
are not available for all bunches. In practice one of BRIL luminometers has to be used instead of PCC. The most linear BRIL luminometer relative to PCC was defined from $\mu$ scan performed in the special fill consisted of isolated bunches ${ }^{3}$. Therefore, the influence of signal from a bunch to the signal from next bunches is negligible. As shown in Fig. [3] BCM1FSI has the lowest $p_{1}$ and, therefore, it is used as the reference luminometer $\mathrm{L}_{\text {ref }}$.

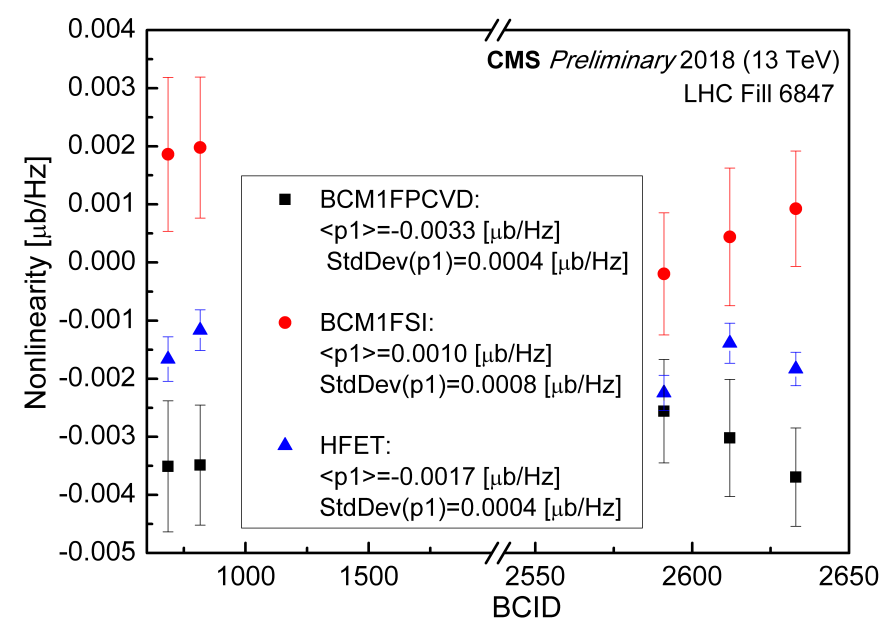

Figure 3: Per-BCID nonlinearity of BRIL luminometers relative to PCC. Data from $\mu$ scan performed in LHC fill 6847 with isolated bunches. PCC data are available for five BCIDs. The mean value of nonlinearity over BCIDs and its standard deviation ("StdDev") are shown in the legend. The mean nonlinearity for HFOC is $-0.0010 \mu \mathrm{b} / \mathrm{Hz}$ with a standard deviation of $0.0005 \mu \mathrm{b} / \mathrm{Hz}$ (not shown in the plot).

Let us consider the three luminometers $\mathrm{L}_{1}, \mathrm{~L}_{2}, \mathrm{~L}_{3}$ and the resulting nonlinearities: nonlinearity of $\mathrm{L}_{1}$ with $\mathrm{L}_{\text {ref }}=\mathrm{L}_{2}\left(p_{1}^{\prime}\left(\mathrm{L}_{1}\right)\right)$, nonlinearity of $\mathrm{L}_{1}$ with $\mathrm{L}_{\text {ref }}=\mathrm{L}_{3}\left(p_{1}\left(\mathrm{~L}_{1}\right)\right)$ and nonlinearity of $\mathrm{L}_{2}$ with $\mathrm{L}_{\mathrm{ref}}=\mathrm{L}_{3}\left(p_{1}\left(\mathrm{~L}_{2}\right)\right)$. From the definition of nonlinearity and at the assumption that nonlinearity is small, one can obtain the relation

$$
p_{1}^{\prime}\left(\mathrm{L}_{1}\right)=p_{0}\left(\mathrm{~L}_{2}\right) \times p_{1}\left(\mathrm{~L}_{1}\right)+p_{0}\left(\mathrm{~L}_{1}\right) \times p_{1}\left(\mathrm{~L}_{2}\right)
$$

per BCID $\left(p_{0}\left(\mathrm{~L}_{2}\right), p_{0}\left(\mathrm{~L}_{1}\right)\right.$ are fit parameters (2.2) corresponding to $\left.p_{1}\left(\mathrm{~L}_{2}\right), p_{1}\left(\mathrm{~L}_{1}\right)\right)$. For mean values over BCID one can expect

$$
\left\langle p_{1}^{\prime}\left(\mathrm{L}_{1}\right)\right\rangle \approx\left\langle p_{1}\left(\mathrm{~L}_{1}\right)\right\rangle+\left\langle p_{1}\left(\mathrm{~L}_{2}\right)\right\rangle .
$$

Relations (B.]), (B.2) link nonlinearities derived at different reference luminometers.

Nonlinearity when BCM1FSI is used as reference luminometer is shown in Table $\square$ for $\mu$ scans performed in the fill with isolated bunches and in the fill with trains (LHC fills 6847 and 6854, correspondingly). It is seen from the relation (3.2) the mean values for fill 6847 are in agreement

\footnotetext{
${ }^{3}$ The LHC orbit is divided in 3564 bunch slots (numbered with bunch crossing ID, BCID) of 25 ns length. Some of slots can be filled by particles, some can be empty. The sequence of filled BCIDs is called "trains". In the text, the fill when filled BCIDs are surrounded by wide gaps of empty BCIDs is called "fill with isolated bunches". Data-taking fills are usually fills with trains of different configurations.
} 


\begin{tabular}{|l|rr|rr|}
\hline Main BCID pattern: & \multicolumn{2}{|c|}{ Isolated BCIDs } & \multicolumn{2}{|c|}{$136 \mathrm{e}+48 \mathrm{~b}+7 \mathrm{e}+48 \mathrm{~b}+7 \mathrm{e}+48 \mathrm{~b}$} \\
LHC fill number: & \multicolumn{2}{|c|}{6847} & \multicolumn{2}{|c|}{6854} \\
\hline & $\left\langle p_{1}\right\rangle$ & StdDev & $\left\langle p_{1}\right\rangle$ & StdDev \\
\hline SBIL(PCVD)/SBIL(SI) & -0.004 & 0.0009 & -0.0069 & 0.0018 \\
SBIL(HFET)/SBIL(SI) & -0.0025 & 0.0008 & -0.0009 & 0.0012 \\
SBIL(HFOC)/SBIL(SI) & -0.0017 & 0.0008 & 0.0031 & 0.0014 \\
\hline
\end{tabular}

Table 1: Average over BCID slope $\left\langle p_{1}\right\rangle$ (mean nonlinearity) and its standard deviation (in $\mu \mathrm{b} / \mathrm{Hz}$ ) derived from $\mu$ scans for BRIL luminometers BCM1FPCVD, HFOC, and HFET relative to BCM1FSI. In upper right cell: e — empty BCID, b — filled BCID.

with values shown in Fig. B. For fill 6854 nonlinearity per BCID for three trains is shown in Fig. 田. The mean values for fill 6854 differ significantly from the ones for fill 6847 . Therefore, the nonlinearity is strongly dependent on the pattern of filled and empty BCIDs (this pattern is usually called "filling scheme"). That behavior of relative nonlinearity requires additional studies that are outside the scope of this proceedings.

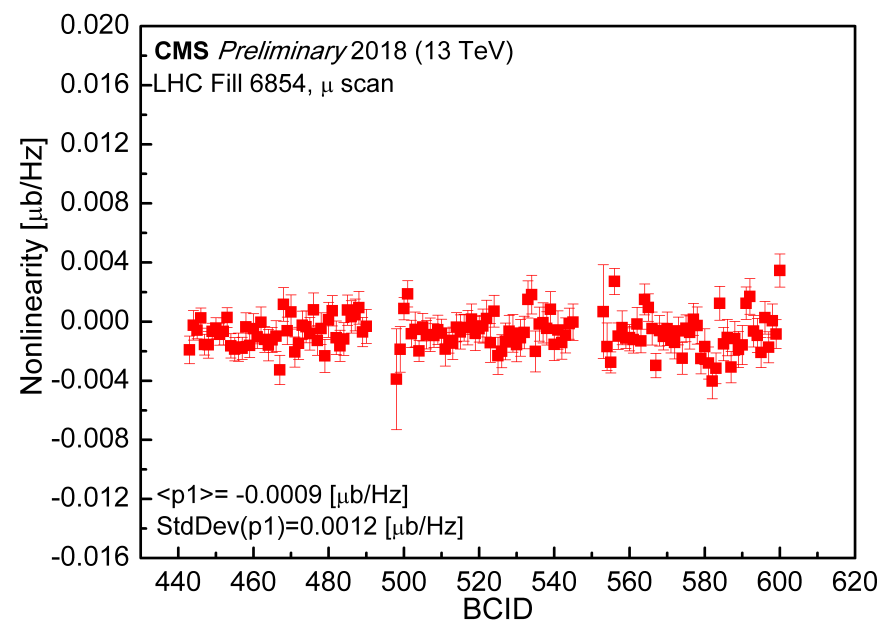

Figure 4: Per-BCID nonlinearity of HFET relative to BCM1FSI. Data are from $\mu$ scan perfomed in fill 6854, trains 5-7. The mean value of nonlinearity and its standard deviation shown in the legend are calculated using all BCIDs in this fill.

\section{Conclusion}

In these proceedings the formalism of relative nonlinearity is developed. Nonlinearity defines the difference between measurements of test luminometer and the response of linear luminometer. If the nonlinearity $p_{1}$ is known the contribution of the first nonlinear term to the response function is $p_{1} \times \mathrm{SBIL}^{2}$ and, therefore, the corresponding correction could be applied: $\mathrm{SBIL}_{\mathrm{corr}}=\mathrm{SBIL}-$ $p_{1} \times \mathrm{SBIL}^{2}$. Here $\mathrm{SBIL}_{\text {corr }}$ is the corrected luminosity without the contribution from the nonlinear term. It corresponds to the luminosity measured by the luminometer with linear response. From 
Table $\mathrm{W} p_{1}$ is of the order of $10^{-3}-10^{-4}$. In the studied $\mu$ scans the highest SBIL is about $8 \mathrm{~Hz} / \mu \mathrm{b}$. Therefore, even if $p_{1}$ is so small the contribution from the nonlinear term could be up to one percent. At future HL-LHC conditions SBIL up to $20 \mathrm{~Hz} / \mu \mathrm{b}$ is expected and even such a small nonlinearity can lead to $1.5-2.0 \%$ effect on luminosity. Nonlinearity studies will be very important to understand the performance of new luminometers designed to operate at HL-LHC conditions.

\section{References}

[1] CMS Collaboration, The CMS experiment at the CERN LHC, DINST 3(2008) SO8004.

[2] A. Penzo and Y. Onel, The CMS-HF quartz fiber calorimeters, J. Phys. Conf. Ser. 160 (2009) 012014.

[3] B. Bilki, Comissioning of Upgrade Forward Hadron Calorimeters of CMS, in proceedings of 2016 IEEE NSS/MIC/RTSD (2016).

[4] J. L. Leonard et al., Fast Beam Condition Monitor for CMS: perfomance and upgrade, Nucl. Instrum. and Meth. A 765 (2014) 235.

[5] CMS Collaboration, CMS luminosity measurement for the 2017 data-taking period at $\sqrt{s}=13 \mathrm{TeV}$, CMS PAS LUM-17-004 (2018).

[6] P. Grafström and W. Kozanecki, Luminosity determination at proton colliders, Prog. Part. Nucl. Phys. 81 (2015) 97.

[7] O.S. Brüning et al., LHC design report, CERN, Geneva 2004. 